Jutta Albus | Kirsten Hollmann-Schröter | Felix Lowin | Matthäus Johann Nowak

SYSTEMATISIERTE

PLANUNGS- UND BAUPROZESSE

Hintergründe, Strategien und Potenziale industrieller Vorfertigungstechnologien

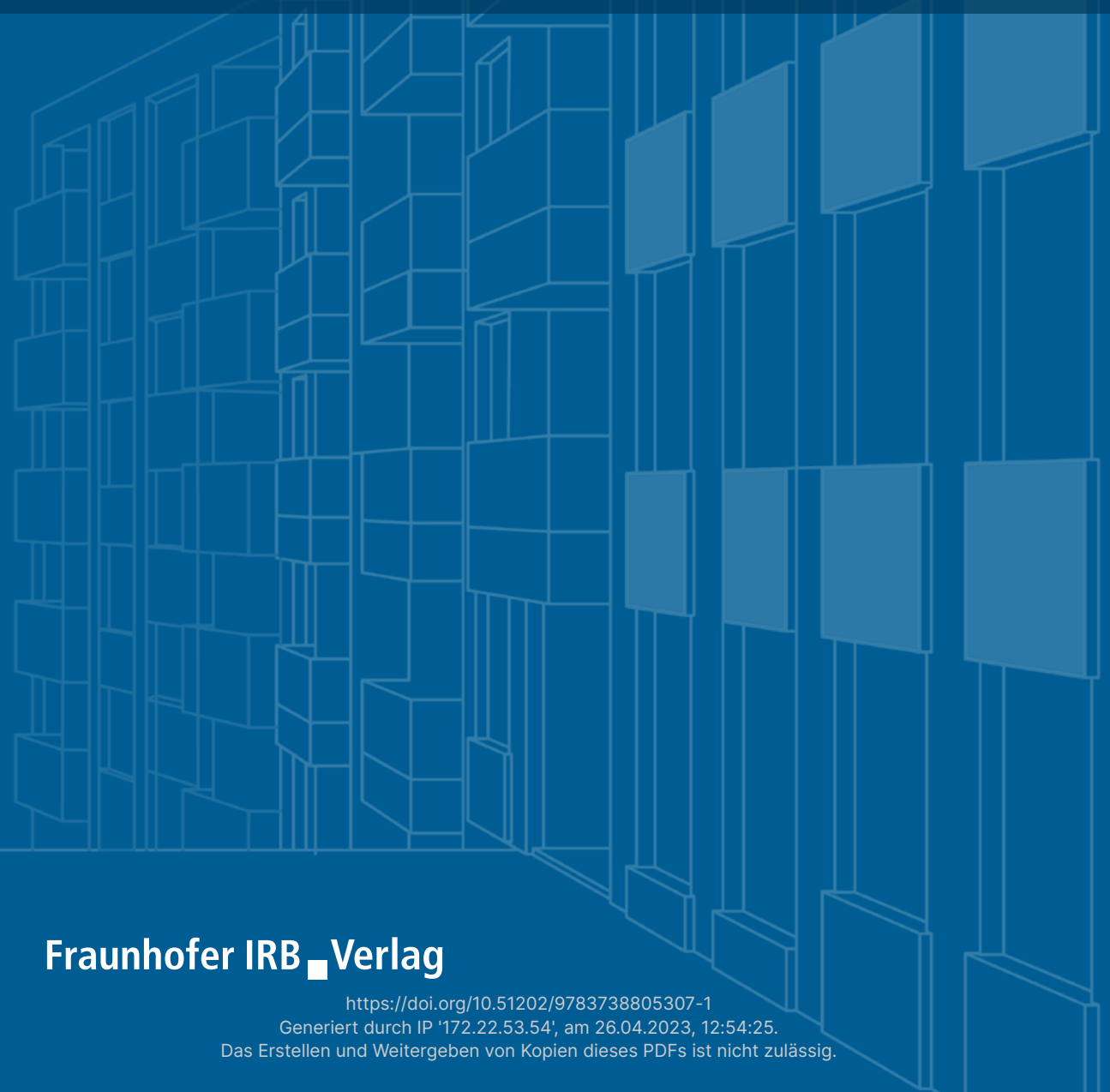


Jutta Albus

Kirsten Hollmann-Schröter

Felix Lowin

Matthäus Johann Nowak

Systematisierte Planungs- und Bauprozesse 


\section{Jutta Albus}

Kirsten Hollmann-Schröter

Felix Lowin

Matthäus Johann Nowak

\section{SYSTEMATISIERTE PLANUNGS- UND BAUPROZESSE}

Hintergründe, Strategien und Potenziale industrieller

Vorfertigungstechnologien 
Bibliografische Information der Deutschen

Nationalbibliothek:

Die Deutsche Nationalbibliothek verzeichnet diese

Publikation in der Deutschen Nationalbibliografie;

detaillierte bibliografische Daten sind im Internet über

www.dnb.de abrufbar.

ISBN (Print): 978-3-7388-0529-1

ISBN (E-Book): 978-3-7388-0530-7

Satz, Layout, Herstellung: Andreas Preising

Umschlaggestaltung: Martin Kjer

Druck: W. Kohlhammer Druckerei GmbH + Co. KG, Stuttgart

(C) Fraunhofer IRB Verlag, 2021

Fraunhofer-Informationszentrum Raum und Bau IRB

Nobelstraße 12, 70569 Stuttgart

Telefon +49 711 970-2500

Telefax +49 711 970-2508

irb@irb.fraunhofer.de

www.baufachinformation.de
Die hier zitierten Normen sind mit Erlaubnis des DIN Deutsches Institut für Normung e.V. wiedergegeben. Maßgebend für das Anwenden einer Norm ist deren Fassung mit dem neuesten Ausgabedatum, die bei der Beuth Verlag GmbH, Burggrafenstraße 6, 10787 Berlin, erhältlich ist.

Alle Rechte vorbehalten.

Dieses Werk ist einschließlich aller seiner Teile urheberrechtlich geschützt. Jede Verwertung, die über die engen Grenzen des Urheberrechtsgesetzes hinausgeht, ist ohne schriftliche Zustimmung des Fraunhofer IRB Verlages unzulässig und strafbar. Dies gilt insbesondere für Vervielfältigungen, Übersetzungen, Mikroverfilmungen sowie die Speicherung in elektronischen Systemen.

Die Wiedergabe von Warenbezeichnungen und Handelsnamen in diesem Buch berechtigt nicht zu der Annahme, dass solche Bezeichnungen im Sinne der Warenzeichen- und Markenschutz-Gesetzgebung als frei zu betrachten wären und deshalb von jedermann benutzt werden dürften.

Sollte in diesem Werk direkt oder indirekt auf Gesetze, Vorschriften oder Richtlinien (z.B. DIN, VDI, VDE) Bezug genommen oder aus innen zitiert werden, kann der Verlag keine Gewähr für Richtigkeit, Vollständigkeit oder Aktualität übernehmen. Es empfiehlt sich, gegebenenfalls für die eigenen Arbeiten die vollständigen Vorschriften oder Richtlinien in der jeweils gültigen Fassung hinzuzuziehen. 


\section{Inhalt}

Einleitung

1. Einführung

1.1 Vorfertigung, Systematisierte

Bauweisen und industrielle Produktion _ 11

1.1.1 Industrielles Bauen

1.1.2 Vorfertigung

1.1.3 Systematisierung, Typisierung und System

1.1.4 Serielles Bauen

1.2 Warum eignen sich Vorfertigung und systematisierte Bauweisen für einen Einsatz in der Architektur und im Bauwesen? 18

1.3 Welche Treiber gab es (in der Vergangenheit) und welche Konsequenzen hatten diese auf heutige Entwicklungen? 20

1.3.1 Impuls - Manifestation - Effekt 20

1.3.2 Das erste Haus oder der Mensch als Nomade

1.3.3 Handwerk und Holzbau 21

1.3.4 Kolonialisierung und Militärwesen

1.3.5 Fortschrittsdenken und die industrielle Revolution 25

1.3.6 Steigerung der Effizienz und Rationalisierung vs. Objektwert

1.3.7 Permanente Anpassung (von Konstruktion und Material) 29

1.3.8 Wohnungsnot und Massenproduktion 32

1.3.9 Monotonie und Maßstab von Großwohnsiedlungen I Nachwirkungen 34

\subsubsection{Faktor Zeit} 51

1.5.4 Änderung der Beziehungsmatrix bei Einsatz von Vorfertigung 52

1.5.5 Dynamisches Wechselspiel - was erwarten wir in Zukunft 55

2. Planungsbeteiligte, Anforderungen und Einflussfaktoren 57

2.1 Marktakzeptanz - Planer*innen und Baubeteiligte vs. Nutzer*innen 57

2.1.1 Marktakzeptanz - Status Quo 57

2.1.2 Potenziale vs. Vorbehalte 58

2.1.3 Marktakzeptanz - Planer*innen und Baubeteiligte

2.1.4 Vereinbarkeit von Anwendungsvielfalt und Systemgedanken

2.1.5 Projektbezogene Vorfertigung vs. industrielle Massenproduktion

2.1.6 Gestaltungsmöglichkeiten von Bausystemen 64

2.1.7 Marktakzeptanz - Nutzer*innen 65 
2.1.9 Fertighaus I Plattenbau 67

2.1.10 Faktoren zur Erhöhung der Akzeptanz 69

2.1.11 Individualität (vs. Masse) I Nutzerspezifische Maßanfertigung

2.1.12 Varianz in Serie (vs. Monotonie)

2.1.13 Offene Systeme

2.1.14 Soziale Durchmischung

2.1.15 Identifikation

2.1.16 Integration I Partizipation

2.2 Vergaberichtlinien

2.2.1 Konventioneller Bauablauf und einhergehende Vergabepraxis

2.2.2 Anforderungen an den Einsatz von vorgefertigten Bausystemen

2.2.3 Mögliche Vergabemodelle

2.2.4 Was kommt nach der HOAl? Chancen für innovative Bauprozesse

2.3 Anforderungen an Planung und Bauwerk

2.3.1 Grundlagen- bzw. Bedarfsermittlung

2.3.2 Anforderungen der Beteiligten

2.3.3 Technische Anforderungen

2.3.4 Die Kunst der Fuge

3. Stand der Technik 101

3.1 Überblick über systematisierte Bauweisen und deren Einsatzmöglichkeiten 102

\subsection{Materialien und} Konstruktionstypologien 103

3.2.1 Konstruktionstypologie 103

3.2.2 Holzbau - Werkstoff und Konstruktion 106

3.2.3 Stahlbauweisen 122

3.2.4 Massive Bauweisen 136

3.3 Produktion, Montage und Transport _ 147

3.3.1 Produktion 148

3.3.2 Logistik, Transport und Montage

3.4 Kostenbetrachtung 151

3.4.1 Kostendarstellung Wohnungsbau 153

3.4.2 Aufzeigen von Potenzialen 154 157
4. Innovation durch neue Planungswerkzeuge und Vorfertigungstechnologien 161

4.1 Maßnahmen und mögliche Planungsstrategien 163

4.1.1 Optimierung von Planung und Produktion durch computerbasierte Werkzeuge 163

4.1.2 Veränderungen an der Schnittstelle ___ 165

4.1.3 Einsatz von innovativen Technologien in der Produktion 169

4.2 Effizienzstrategien und Gestaltungsanspruch 177

4.2.1 Intelligente Planungskonzepte zur Steigerung der Effizienz und Varianz 178

4.2.2 Systemgedanke und Gestaltungsvielfalt _ 190

5. Annex 197

5.1 HAUT, Amsterdam 197

5.1.1 Programm und Funktion _ 197

5.1.2 Bauweise und Konstruktion _ 198

5.1.3 Material und Fertigung __ 199

5.1.4 Systemgedanke und Gestaltungsvielfalt _ 200

5.2 Mehrgenerationenhaus, Wuppertal___201

5.2.1 Programm und Funktion __ 201

5.2.2 Bauweise und Konstruktion __ 202

5.2.3 Material und Fertigung __ 203

5.2.4 Systemgedanke und Gestaltungsvielfalt _ 204

$5.3 \mathrm{HoHo}$, Wien 205

5.3.1 Programm und Funktion__ 205

5.3.2 Bauweise und Konstruktion _ 206

5.3.3 Material und Fertigung _ 207

5.3.4 Systemgedanke und Gestaltungsvielfalt _ 208

Schlusswort 209

Literatur 211

Stichwortregister 215 